\title{
Exploring Smart Home and Internet of Things Technologies for Smart Public Buildings
}

\author{
Myriam Guedey \\ Dieter Uckelmann* \\ myriam.guedey@hft-stuttgart.de \\ dieter.uckelmann@hft-stuttgart.de \\ Information Logistics \\ University of Applied Sciences Stuttgart \\ Stuttgart, Germany
}

\begin{abstract}
Recent years have seen a rapid increase in the development and use of smart home technologies. Besides being used in private homes, these and other wireless Internet of Things (IoT) technologies may also complement classical building automation to retrofit existing stock. Especially public and commercial buildings in Germany and Europe have the potential to become more energy-efficient and to provide a healthier environment for their users. This paper introduces an ongoing feasibility study on Smart Public Buildings at the University of Applied Sciences Stuttgart. The study addresses the special requirements of public institutions for the utilization of smart home and other IoT technologies. To explore and validate these requirements, different use cases and prototypes based on an open-source platform for the home automation have been developed. Firstly, a preceding analysis of the special requirements of public buildings in Germany is outlined. Secondly, the basic installation of a smart building system at the university campus is introduced. Finally, some of the prototypes and use cases that have been carried out are presented.
\end{abstract}

\section{CCS CONCEPTS}

- Computer systems organization $\rightarrow$ Sensor networks; $\bullet$ Hardware $\rightarrow$ Wireless integrated network sensors; Sensor devices and platforms.

\section{KEYWORDS}

smart (public) buildings, smart home, open-source software, building automation

\section{ACM Reference Format:}

Myriam Guedey and Dieter Uckelmann. 2020. Exploring Smart Home and Internet of Things Technologies for Smart Public Buildings. In 10th International Conference on the Internet of Things (IoT '20), October 6-9, 2020, Malmö, Sweden. ACM, New York, NY, USA, 8 pages. https://doi.org/10.1145/3410992. 3411009

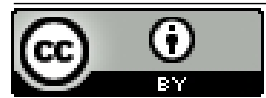

This work is licensed under a Creative Commons Attribution International 4.0 License.

IoT '20, October 6-9, 2020, Malmö, Sweden

(C) 2020 Copyright held by the owner/author(s).

ACM ISBN 978-1-4503-8758-3/20/10.

https://doi.org/10.1145/3410992.3411009

\section{INTRODUCTION}

In Europe, around 40 percent of energy consumption and 36 percent of $\mathrm{CO} 2$ emissions are related to buildings [12]. A big share of these buildings is older than 50 years, built before the first thermal regulations and any kind of smart, networked system emerged. While residential buildings can benefit from the vast development of smart home appliances to become more energy-efficient and comfortable, the use of wireless IoT technologies in non-residential buildings, especially in public buildings, has been cautious so far. The advantages of wireless sensors and actuators, such as lower investment costs and the elimination of wiring walls, come with some downsides - first of all, the huge heterogeneity of technical solutions in the field and thus incompatibility issues and complex installation procedures.

Furthermore, the smart home market is fragmented and volatile, many different vendors exist and current solutions might be obsolete within five years. Often they are designed as closed systems, tied to proprietary hard- and software, relying on arbitrary cloud services. This kind of design comes with a loss of control over data for the operators of such systems. All these indications run contrary to recommendations for public institutions $[15,18,29]$ that point out the importance of striving at vendor and technology independence. Solutions based on open-source software are regarded as a possible way to avoid the so-called vendor lock-in [15, 22].

The presented analysis and use cases are part of ongoing research on Smart Public Buildings at the University of Applied Sciences Stuttgart (HFT Stuttgart). It explores the opportunities for retrofitting public buildings with smart home and other IoT technologies to reduce energy consumption and $\mathrm{CO} 2$ emissions as well as to increase occupancy rate and user comfort. The methodological approach is based on the design and prototypical implementation of various use cases within the university's buildings to validate the technical feasibility. The prototypes build on an open-source platform for the smart home that manages devices and services technology- and vendor-independent [3]. Exemplary, the open Home Automation Bus (openHAB) [24] is used. Research questions aim at a) the special requirements of public buildings regarding smart systems and technologies, b) the suitability of different smart home and IoT technologies for public buildings, c) the opportunities that public buildings provide for the application of those technologies and d) the impact of smart public buildings on data privacy. 


\section{PUBLIC BUILDINGS IN GERMANY}

Public buildings differ from residential buildings in many respects. A preceding analysis of characteristics has been carried out to derive special requirements of public institutions [14] regarding the application of smart home and other IoT technologies. Although there is no common, precise definition of a public building within the EU - definitions range from "are not apartments or are nonresidential” (Czech Republic), "provide public services” (Finland) to "occupied by a governmental body" (France) [6] - the German DIN standard refers to public buildings as "publicly accessible buildings / intended for public use" and classifies them in "cultural and educational institutions, sports and recreational facilities, health care facilities, office, administrative and courthouse buildings, shops and restaurants, parking lots, garages, and toilets" [7]. Based on this definition we have derived the following characteristics to distinguish typical residential from public buildings in Germany (see Table 1):

Table 1: Characteristics of buildings in Germany

\begin{tabular}{lll}
\hline Type & Residential & Public \\
\hline Access & Few & Everyone \\
\hline Usage & Dwelling & Diverse \\
\hline Size (m2), Ø & $91.8[27]$ & 900 (Stuttgart) [14] \\
\hline Users/day, Ø & $2[28]$ & 300 (Stuttgart) [14] \\
\hline Privacy & Not applicable & Mandatory \\
\hline
\end{tabular}

Access: A public building is accessible by the public. Though this can be narrowed down to subgroups - for example only employees having access to certain rooms - public in general implies a quite large group of authorized users, whereas usually, only a few people have access to a private home.

Usage: While private homes are comparable regarding their typical room functions (e.g. kitchen, bathroom, bedroom, living room), the general purpose of public buildings appears to be more diverse, ranging from education (school, library), sports (swimming bath) or culture (museum, theatre) to administration (town hall) and health (hospitals).

Size: In general, public buildings are much larger than residential buildings or apartments. According to a questionnaire from 2017 (sample size $=50$ ), public buildings in the city of Stuttgart, Germany, have a size of 900 square meters on average [14]. In comparison to that, the average dwelling space in Germany measures 91.8 square meters [27].

Users: Public buildings often serve as a place where people gather: travelers in the station building, the city council in the boardroom, exhibition visitors in the museum. In Stuttgart, public buildings are frequented by around 300 visitors per day, whereas private homes in Germany count only 2 inhabitants on average [28]. Furthermore, public buildings are more often frequented by changing users than residential buildings on a day to day basis.

Privacy: Since May 2018, the European General Data Protection Regulation (GDPR) applies to every company or entity that processes and/or holds personal data (with relation to the EU) [13]. The GDPR does not apply to private homes and households. This concerns, for example, the use of video surveillance: whereas in private homes one may use cameras to monitor occupancy, this is not the case in public buildings - especially video surveillance must be justified and weighed against the users' rights.

\subsection{Smart Public Buildings}

The term building automation is originally used to describe the automation of facilities and systems closely connected to the building (heating, ventilation, air-conditioning - HVAC), and is typically based on wired networks using specific protocols, such as KNX, BACnet, and Modbus [32]. A smart building furthermore includes systems such as access control, fire alarms, video monitoring, and multi-media and is focusing also on user's comfort and security [26]. Technologies in a smart building more often connect via radio transmission and protocols from the Internet of Things (Wi-Fi, Bluetooth, ZigBee, ZWave, LoRaWAN, etc.) and capabilities may also include intelligent planning under consideration of building state and user needs $[16,31]$.

Introducing the term Smart Public Building (SPB), we refer to public buildings retrofitted with wireless technologies and a building management system for monitoring and automating building functions to address the needs of multiple stakeholders. From the characteristics discussed in section 2, we have derived special requirements of public buildings regarding smart building systems. Table 2 describes some of these requirements and lists corresponding prototypes and use cases presented in section 3 and 4 .

\section{TESTBED UNIVERSITY CAMPUS}

The University of Applied Sciences Stuttgart is located in the city center. The campus consists of seven buildings (Figure 1) from different years of construction, ranging from 2016 back to the $19^{\text {th }}$ century. The university is the tenant of most of the buildings, sharing some with other occupants - public as well as commercial facilities. As stated in section 2, usage of public buildings is diverse and at HFT this includes assembly rooms, lecture rooms, student workplaces, staff offices, library, workshops, laboratories, cafeteria, computer center, parking areas, and courtyards.

Building 2, one of the older university buildings, has been selected as a test bed for the research. HFT has leased this building and is the only occupant. Besides staff offices, it hosts lecture rooms and computer labs on each floor as well as some workshops in the basement. In total, the building spans seven floors with approximately 7,000 square meters. No monitoring or automation system has been installed before this research.

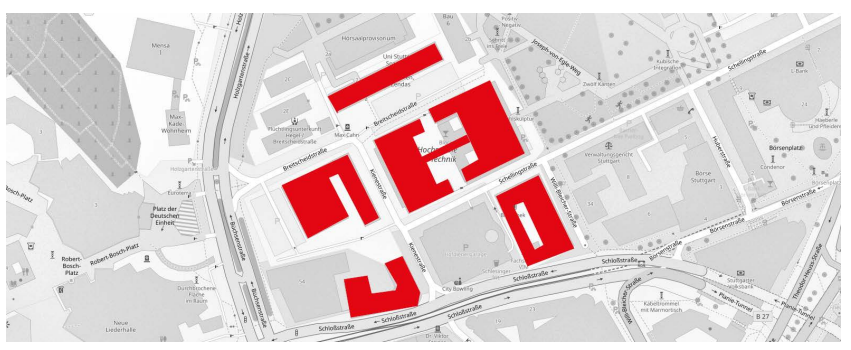

Figure 1: Campus of HFT Stuttgart (map: OpenStreetMap) 
Table 2: Requirements of public buildings (scope: Germany)

\begin{tabular}{|c|c|c|}
\hline Requirement & Examples & Corresponding use case/prototype \\
\hline \multicolumn{3}{|l|}{ Access } \\
\hline Safety & $\begin{array}{l}\text { Evacuation in case of an emergency (escape routes, fire } \\
\text { doors), availability of safety-relevant equipment (fire } \\
\text { extinguishers, defibrillators). }\end{array}$ & $\begin{array}{l}\text { Monitoring Safety-relevant Devices (not presented) } \\
\text { Visualization for Building Control (not presented) }\end{array}$ \\
\hline \multicolumn{3}{|l|}{ Usage } \\
\hline Reliability & $\begin{array}{l}\text { Stable running systems, security of the network, of } \\
\text { devices and software components. }\end{array}$ & $\begin{array}{l}\text { 3.3 Smart Distributed Systems } \\
\text { Location Based Services with Beacons (not presented) }\end{array}$ \\
\hline Terms of use & $\begin{array}{l}\text { House rules (referring to opening hours, handling of } \\
\text { equipment, closing windows and doors, damage re- } \\
\text { porting, etc.) [20]. }\end{array}$ & $\begin{array}{l}\text { 4.3 Smart Lecture Room } \\
\text { Damage Reporting in Public Buildings (not presented) }\end{array}$ \\
\hline \multicolumn{3}{|c|}{ C } \\
\hline Devices & $\begin{array}{l}\text { Necessity of more sensors, actuators, gateways, etc., } \\
\text { integration of additional systems (e.g. elevators, utility } \\
\text { meters and parking lots). }\end{array}$ & $\begin{array}{l}\text { 4.1 Smart Metering } \\
\text { 4.3 Smart Lecture Room } \\
\text { Location Based Services with Beacons (not presented) }\end{array}$ \\
\hline Connectivity & $\begin{array}{l}\text { Wireless services have to cover larger areas, taking } \\
\text { into account that radio systems are more susceptible } \\
\text { to interference. }\end{array}$ & $\begin{array}{l}\text { 4.1 Smart Metering } \\
\text { 3.3 Smart Distributed Systems } \\
\text { Monitoring Safety-relevant Devices (not presented) }\end{array}$ \\
\hline \multicolumn{3}{|l|}{ Users } \\
\hline Number & $\begin{array}{l}\text { Monitoring actual occupancy of specific rooms and } \\
\text { areas, providing smart building services according to } \\
\text { the need of many different users. }\end{array}$ & $\begin{array}{l}\text { 4.2 Room Occupancy } \\
\text { 4.3 Smart Lecture Room } \\
\text { Building Automation via User Polling (not presented) }\end{array}$ \\
\hline \multicolumn{3}{|l|}{ Privacy } \\
\hline GDPR compliance & $\begin{array}{l}\text { Video surveillance to provide comfort functions may } \\
\text { be feasible in a private home. By implication, this does } \\
\text { not apply to a public building. }\end{array}$ & $\begin{array}{l}\text { 4.2 Room Occupancy } \\
\text { 4.3 Smart Lecture Room } \\
\text { Building Automation via User Polling (not presented) }\end{array}$ \\
\hline
\end{tabular}

\subsection{An Open Source Ecosystem}

As stated in the introduction, open-source software is proposed as a possible solution for public institutions to avoid vendor lock-in. This also reflects the recommendations of the Smart City Charta by the German Federal Institute for Research on Building, Urban Affairs and Spatial Development [18].

OpenHAB (open Home Automation Bus) is one of the currently available, mature open-source software projects for the smart home [24]. The software acts as a hub, integrating different smart devices, technologies, and services into a single platform. Due to its open and modular approach based on the OSGi framework, openHAB can be adapted to specific use cases. The project has a large, active user and developer community, which can be regarded as an indicator for sustainability [1]. Another popular open-source smart home platform is Home Assistant, though its short release cycle with regular major breaking changes [21] runs contrary to the stated requirements of public buildings (Table 2, "Reliability").

A common openHAB setup consists of additional software components. These components provide further functionality, such as persisting data, managing telemetry data transport, and adding a network security layer. The typical openHAB stack [8] can be built completely on open-source software, as listed in the following subsection. According to a non-representative community survey from 2019 [9], 74 percent of openHAB users maintain a single open$\mathrm{HAB}$ instance, 98 percent use it to monitor and control a private house or apartment and 99 percent run openHAB with less than ten people having access to it. These results indicate, that maintaining openHAB in a large public building or even in multiple large public buildings, like a campus, with a higher number of users can be considered as a new field of application.

\subsection{Basic Installation in Building 2}

A client-server system serves as the basis for the different use cases and prototypes. Up to now, the system spreads over five floors in building 2, connecting around 120 wireless sensors and actuators to the server. The sensors, actuators, and gateways have been deployed in offices, lecture rooms (section 4.3), and computer labs. The installation furthermore includes utility meters in the basement (section 4.1). On the server side, the following software components and services have been installed and configured:

openHAB: The current stable release serves as an interactive management platform, connecting sensors, actuators, and other sources of data. Several user interfaces allow the management of the installation as well as the information of users.

InfluxDB: Single and aggregated IoT data are persisted in an InfluxDB as well as in a MySQL database (for historical reasons). The saving interval can be defined in openHAB, and may be time or event-based.

Node-RED: The graphical development environment based on JavaScript is used to manage and process some of the data flows from external components to openHAB. 
Grafana: A monitoring tool for visualizing and analyzing time series of data. Having access to the database, different kinds of charts can be generated with Grafana and embedded into user interfaces of openHAB.

Mosquitto: A message broker for the MQTT protocol that allows the connection of devices not yet supported by openHAB (see sections 4.1,4.2). Those devices may publish their data on a broker's channel, whereas an openHAB item, the logical representation of a device's functionality, subscribes to that channel.

Nginx: By default, openHAB is exposed to the local network. The reverse proxy Nginx provides a way of securing the openHAB server with authentication and Transport Layer Security.

The above-described installation is intended to cover not only different floors in building 2 but also more buildings on the campus. Therefore, a concept and a prototype for managing distributed smart systems based on openHAB have been developed.

\subsection{Distributed Smart Systems}

As outlined in section 2, public buildings on average are significantly larger than apartments or residential buildings. Moreover, the university campus consists of multiple large buildings. Thus, several technical aspects have to be considered when deploying smart home and other IoT devices and software in a public institution like a university.

Not only a higher number of devices is required to retrofit a large building but also a larger area has to be covered by the wireless service. Either which wireless technologies are used (e.g. ZigBee, ZWave, EnOcean), it is likely that more than one hub (gateway) per technology will be necessary to provide a large building with connectivity (cf. Table 2, "Connectivity"). This is due to limited transmission ranges and specific gateway capabilities, such as the maximum number of addressable devices. Hence follows another setup than typically found in smart homes, where one server acting as the central hub for all wireless devices may be sufficient (Figure 2) $[9,19]$.
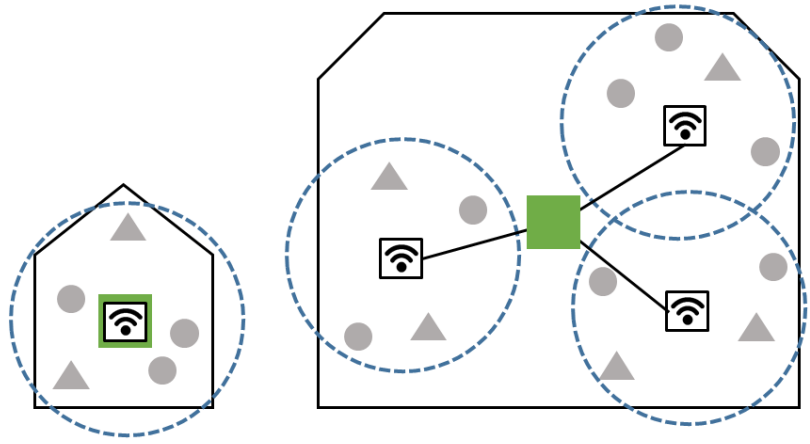

openHAB server

sensors, actuators

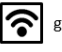

gateway (transceiver)

Figure 2: Distributed system for large buildings compared to a common smart home system, based on [19]

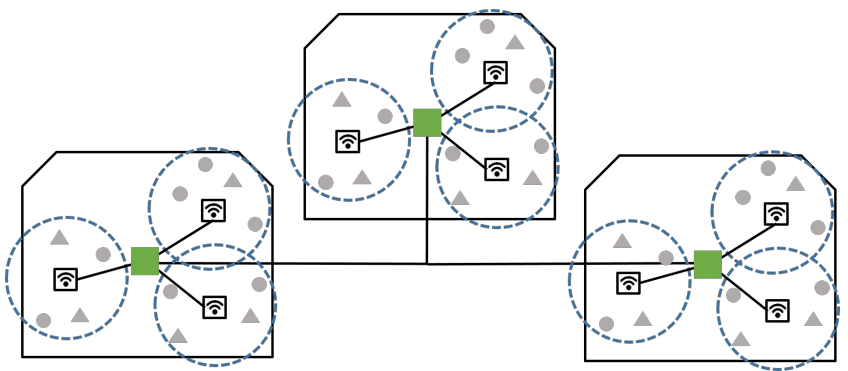

Figure 3: Distributed system for multiple (large) buildings

a) Distributed system for large buildings: The distribution of multiple hubs in a large public building connecting to one openHAB server (Figure 2) has already been the subject of research [19]. An adapted version of the proposed method - connecting a remote hub via MQTT and shadow items to the main openHAB instance has been used to connect devices in the upper floors of building 2 to the server. Nonetheless, to maintain a group of buildings such as a university campus, it may be considered providing more than one openHAB server, e.g. one per building, to ensure independent operability and availability (cf. Table 2, "Reliability").

b) Distributed system for multiple (large) buildings: The prototype developed at HFT Stuttgart focuses on distributed systems spreading over multiple buildings (Figure 3) where each building should be operable independently from each other, but manageable centrally. While the installation and maintenance of openHAB for a single home is fairly simple [25], it can turn out to be timeconsuming and complex in an environment with multiple buildings. Therefore, the goal was to ease the installation and maintenance of the openHAB software stack (cf. 3.2 to ensure a sustainable usage in public buildings.

c) Adaption of openHAB for multiple buildings: Concept and design propose the use of containers to deploy and manage multiple openHAB instances [8]. Containers are sand-boxed environments, that run independently from the applications on the host OS. Furthermore, containers work in different target environments, from virtual machine to Raspberry $\mathrm{Pi}$, thus providing flexibility regarding the existing infrastructures in public institutions, which can vary a lot. Exemplary, Docker is used for containerization. Docker is an open-source software development platform that enables management and deployment of applications running in containers. Within a single configuration file, all services to be installed are defined. These services include the entire openHAB software stack (openHAB, Grafana, InfluxDB, etc.). Docker then handles download and installation of all the applications and their dependencies.

To run multiple instances side by side as well as to orchestrate them, the proposed concept makes use of the container orchestration tool Docker Swarm (Figure 4). In Docker Swarm, the cluster of machines (nodes) that are part of the Smart Public Building system (swarm) can be defined. Nodes can join and leave the swarm and even the replacement of machines, e.g. in case of failure, can be done without much effort (cf. Table 2, "Reliability"). Within the above-mentioned configuration file, it is furthermore set, which services should be deployed to which servers (buildings). 


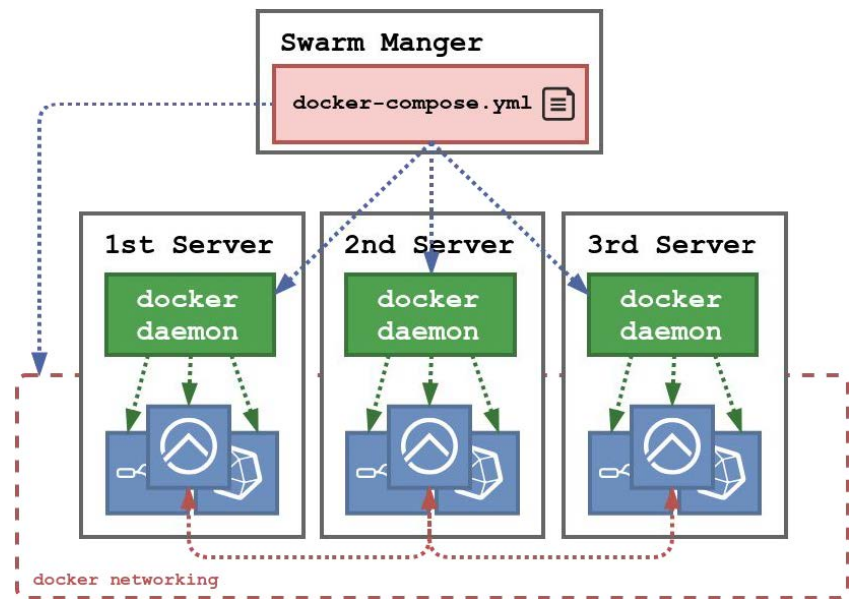

Figure 4: Managing multiple smart building systems, based on [8]

Security is a major concern when implementing smart systems in public institutions. OpenHAB as well as most services that are used along with it by default are easily accessible on the local network. Docker Swarm creates an overlay network that is not accessible by the host system or the local network but allows communication between applications inside the swarm, even across multiple hosts [5]. Furthermore, by adding a reverse proxy like Nginx, it is possible to reduce the exposed ports to only one as well as to provide a basic user authentication (cf. Table 2, "Reliability").

\section{USE CASES AND PROTOTYPES}

Several prototypes and use cases for the application of smart home and other IoT technologies in the university have been developed. They include location-based services, monitoring of safety-relevant devices, social sensors, reporting and managing damages, and others. The implementation of these use cases extends and adapts the basic installation. In doing so, the requirements of public buildings (see Table 2) are further explored and the technical feasibility will be validated. In this section, three of the use cases are introduced: Smart Metering, Room Occupancy, and Smart Lecture Room.

\subsection{Smart Metering}

Since 2014, HFT Stuttgart has been certified according to the EU EcoManagement and Audit Scheme (EMAS). Up to now, responsible technical staff has to read and record the utility meters of every campus building manually, which is done once a month. To provide remote access to energy and water consumption with a higher temporal resolution, the prototype of a monitoring system for the utility meters should be developed and connected to openHAB (cf. Table 2, "Devices"). Regardless of meter type and age, the system should allow access to data in a unified way, enabling analysis and alerts in case of unusual consumption behavior.

a) Connectivity: Three utility meters are installed in building 2 . They are located in the two basement levels, where reliable coverage with the university's Wi-Fi is not given. Using the mobile network on the other hand would have lead to running costs and the need for a power supply per device. Therefore, LoRa, a radio technology operating at the license-free $868 \mathrm{MHz}$ band in Europe, has been chosen to connect the meters (cf. Table 2, "Connectivity"). LoRa was designed for low power consumption and long ranges with good coverage even inside buildings. To provide wireless services with LoRa at the campus, two gateways have been deployed (Figure 5, one in the basement, one at the rooftop of building 3) and connected to The Things Network (TTN) [30], a community-driven open LoRaWAN network.

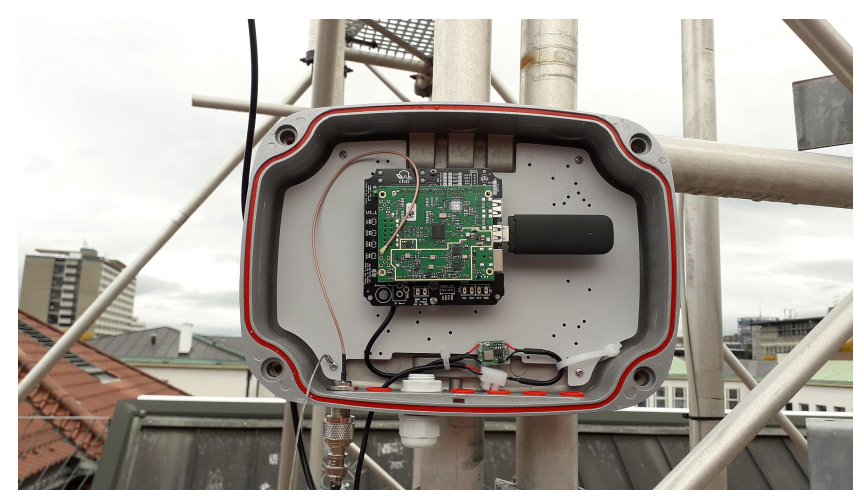

Figure 5: LoRaWAN gateway, rooftop building 3

b) Retrofitting the meters: The utility meters are of different kinds and types (water, heat: Woltman, analog; electricity: phase, digital). Furthermore, three different network operators are in charge of them. One simple technical solution of retrofitting that fits for multiple kinds and types of meters is pulse counting. Each pulse corresponds to a certain amount of energy (kWh) or water (liters). By counting the pulses, it can be determined how much energy or water has been used [23]. Either the meter has already a corresponding pulse output or the responsible operator provides one on request. To count the pulses and transmit the current value, a Pulse Data Capture (PDC) module with LoRaWAN capability has been connected to the meters. At HFT, data are now sent once per hour to The Things Network.

c) Data flow management: TTN doesn't store data received from end devices but provides several possibilities to retrieve it. Originally, MQTT is used to publish the messages. The payload of a message comes as hexadecimal formatted binary data. Thus, several tasks have to be accomplished to integrate the meter data into openHAB: subscribing MQTT messages from TTN, decoding, and processing the received data, pushing the results to the corresponding items (single data endpoints) in openHAB, persisting the data in a database. Latter enables further analysis, visualizations as well as rule-based automation - for example alerting responsible staff if water consumption raises unusually. To manage the described data flow, Node-RED is used.

With the retrofitted meters, it is possible for the facility management, the Eco-management, and researchers at HFT Stuttgart to access utility meter data remotely and at a much finer granulation. 


\subsection{Room Occupancy}

Monitoring actual room occupancy is one of the major topics in many large buildings, especially when the demand for space is growing and an efficient guiding of users is wanted (cf. Table 2, "Number of Users"). Different technologies can be used to detect human presence, such as passive infrared (PIR), image recognition, $\mathrm{CO} 2$ etc. To achieve a simple solution that would not only detect presence (like PIR), but give an estimation of the number of people in a room or a certain open area (no $\mathrm{CO} 2$ ) and also takes privacy into account (in contrast to image recognition), a feasibility study using the Paxcounter has been undertaken.

a) Hard- and software: The Paxcounter [34] is an open-source, ESP32-based prototype with LoRaWAN capability (Figure 6) that counts Wi-Fi and Bluetooth probes of mobile devices according to their Organizationally Unique Identifier (OUI). Originally used in a study of the Deutsche Bahn for passenger flow metering in railway stations, the Paxcounter processes sensitive data (unique identifiers) without intrusion of privacy (cf. Table 2, "GDPR compliance"). No fingerprinting or processing of non-anonymous MAC addresses takes place, and only the bare number of counted devices is transmitted.

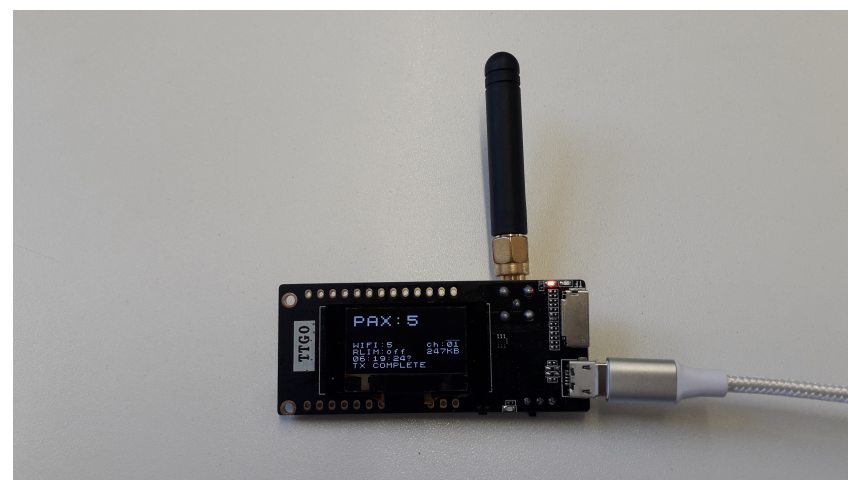

Figure 6: Paxcounter (TTGO v3) with LoRaWAN capability

b) Configuration: Several devices have been installed in lecture and working rooms at HFT Stuttgart. For connectivity and data flow management to openHAB, the same LoRaWAN infrastructure and MQTT bridge as for the Smart Metering have been used. Measurement series have been carried out to find the best configuration for this use case. The series base on different parameter settings concerning Wi-Fi/Bluetooth, signal strength (RSSI) limit, and the length of the cyclic counting interval. The final configuration resulted in a Wi-Fi only counting mode (many mobile devices have Bluetooth deactivated), setting an RSSI limit for each room separately (around $-60 \mathrm{dBm}$ in a medium sized room) to avoid counting too many devices outside the room, and keeping the cycle interval relatively short $(60 \mathrm{sec})$ to not overestimate the number of people.

c) Suitability: Results indicate that the Paxcounter can deliver good hints on whether a room is crowded at a specific time or not, and that a typical usage pattern for certain rooms can be made visible (Figure 7), and accessible to users, e.g. via Grafana and the user interfaces of openHAB. Nonetheless, exact numbers cannot be achieved with the Paxcounter, even not with good parameter adjustments. An impending challenge lies in the trend of mobile operating systems towards using randomly generated, temporary MAC addresses for probe requesting, and even for established connections. Therefore, currently acceptable counting results might become even more inaccurate in the future.

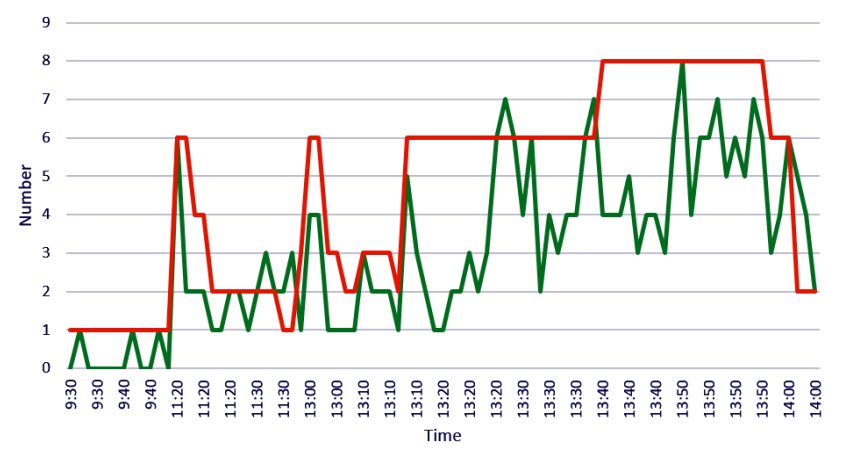

Figure 7: Measurement in a lecture room, RSSI limit $-67 \mathrm{dBm}$. Green: Wi-Fi signals, red: manually counted, based on [10]

\subsection{Smart Lecture Room}

With the application of wireless smart home devices and opensource software, it is possible to retrofit rooms in existing buildings at lower investment costs compared to cabled systems. Besides, wireless technologies can also be used in leased public buildings, allowing extension, relocation, or dismantling of the system with reasonable effort. The Smart Lecture Room describes a concept for monitoring lecture room conditions to optimize energy consumption (heating, lighting) and room occupation as well to provide a more healthy learning environment.

To meet the GDPR (cf. Table 2, "GDPR compliance"), the data protection officer at HFT has been consulted in advance. The Smart Lecture Room as proposed can be considered uncritical regarding GDPR, because collected sensor data do not relate to an identified or identifiable living individual and thus is not considered personal data [11].

Based on the installation described in section 3, several lecture rooms on three floors in building 2 have been retrofitted with different kinds of IoT (electronic-based, software-based, humanbased). Room and outside conditions are constantly monitored and reported to openHAB. Collected data are persisted in the MySQL database and can be used for user information as well as for rulebased room automation and data analysis. So far, the following kinds of IoT have been deployed and integrated:

a) Electronic-based IoT: A multi-sensor is used to measure room conditions such as temperature, humidity, light, and motion. Because all lecture rooms are larger than 20 square meters, several multi-sensors per room have been deployed to cover the area (cf. Table 2, "Devices"). Also, each window has been equipped with a contact sensor to detect the current state (open/closed). Actuators include one thermostat per heater and one plug per multi-media device such as a projector. Table 3 gives an overview of the currently installed sensors and actuators. 
Table 3: Electronic-based IoT - Sensors (S) and Actuators (A)

\begin{tabular}{ll}
\hline Type & Purpose \\
\hline Temperature (S) & $\begin{array}{l}\text { Monitor current temperature to automate } \\
\text { heating dependent on occupancy, optimize } \\
\text { by taking into account outside temperature } \\
\text { as well as forecasts. }\end{array}$ \\
\hline Humidity (S) & $\begin{array}{l}\text { Measure air quality, give feedback to users } \\
\text { on ventilation times when combined with } \\
\text { weather data, alert in case of sudden rise in } \\
\text { measurements. }\end{array}$ \\
\hline Motion (S) & $\begin{array}{l}\text { Adjust room functions based on presence or } \\
\text { absence of users, optimize via combination } \\
\text { with other sensor data such as CO2. }\end{array}$ \\
\hline Light (S) & $\begin{array}{l}\text { Monitor level of light to detect light switched- } \\
\text { on in unoccupied rooms or to give feedback } \\
\text { in case of adverse lighting conditions. }\end{array}$ \\
\hline Windows (S) & $\begin{array}{l}\text { Avoid open heating valves while windows } \\
\text { are opened, report open windows in unoccu- } \\
\text { pied rooms to responsible staff before closing } \\
\text { hours (cf. Table 2, "House rules"). }\end{array}$ \\
\hline Thermostats (A) & $\begin{array}{l}\text { Adjust heating based on actual occupation, } \\
\text { opening hours, schedules and room condi- } \\
\text { tions (e.g. windows, temperature, weather). }\end{array}$ \\
$\begin{array}{l}\text { Measure energy consumption of plugged } \\
\text { devices, detect a running device, switch } \\
\text { attached devices on/off, repeat signals of } \\
\text { meshed network (cf. Table 2, "Connectivity"). }\end{array}$ \\
\hline
\end{tabular}

Table 4: Software-based IoT

\begin{tabular}{ll}
\hline Type & Purpose \\
\hline Schedules & $\begin{array}{l}\text { Connect to lecture plans to provide information for } \\
\text { users and to optimize use of resources, e.g. heat-up } \\
\text { in advance during the cold months. }\end{array}$ \\
\hline Weather & $\begin{array}{l}\text { Integrate information on local weather conditions } \\
\text { and forecasts to complement interior monitoring } \\
\text { (see Table 3). }\end{array}$ \\
\hline Air quality & $\begin{array}{l}\text { Integrate local outside air quality (temperature, } \\
\text { humidity, PM) gathered by "luftdaten.info" (open } \\
\text { data) to optimize recommendations for ventilation. }\end{array}$ \\
\hline
\end{tabular}

b) Software-based IoT: Besides physical devices web services and other sources of data have been connected to openHAB to enable cross-linked analysis and actions. The connections have been established via different modules (Bindings) and linked to items so that incoming data can be processed and persisted. Table 4 lists all currently integrated web services and other IoT data.

c) Human-based IoT: Especially in older or leased buildings, it is not affordable to automate all building functions. E.g., in building 2, windows can't be opened or closed automatically, this has to be done by the users. Furthermore, user feedback on subjective room conditions can be helpful to improve the system. To integrate these factors, a user feedback system has been designed.

d) Visualization and automation: The mentioned MySQL database can be accessed by the visualization tool Grafana to present time series of data within user interfaces, furthermore, the real-time data is used to create different user interfaces (see Figure 8. Historical and real-time data furthermore serve as a source for rule-based automation. So far, some basic heating rules and several maintenance alerts have been defined, e.g. monitoring the LoRaWAN gateways, getting a notification, if devices run out of battery, or go offline due to connectivity issues. System administrators then get notified via smartphone (Telegram Bot) and/or e-mail (cf. Table 2, "Reliability").

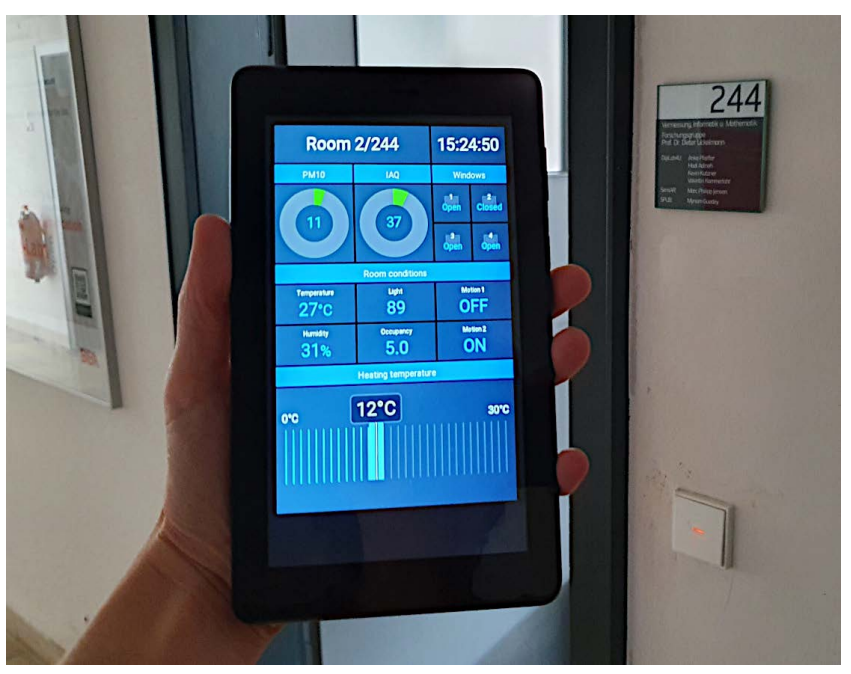

Figure 8: User interface on tablet, room 2/244

\section{CONCLUSIONS AND FUTURE WORK}

In this paper, we propose the application of wireless IoT technologies and smart home open-source software to retrofit public buildings. Many public institutions occupy buildings as tenants, thus facing the same limitations regarding installation possibilities like tenants of private homes. Other public bodies lack funding to retrofit their building stock with cabled automation systems. On the other hand, special requirements of public buildings regarding smart systems pose new challenges towards smart home technologies. The mature open source ecosystem around openHAB allows interoperability and technological independence. We have presented three use cases, outlining that even without adapting the core of openHAB (which is possible, though), different applications for a smart public building can be implemented. If a specific device or service is yet not supported by openHAB, connectivity can be established via the provided REST API or MQTT.

Open challenges lie in possible security issues of smart home technologies [2]. The proposed prototypical extension towards distributed smart systems addresses network security to some extend. It has already been implemented for test purposes and within a 
next step, multiple openHAB servers will be deployed on different machines (buildings) within the university campus.

Future work includes elaboration on the Smart Lecture Room. Further services and devices, such as $\mathrm{CO} 2$ sensors for measuring air quality, are going to be implemented. The rule-based automation will be extended (heating, lighting, general management) as well as complemented by a user feedback system (human-based IoT). In the long run, the lecture rooms should be controlled based on observed usage patterns, thus the connection to data analysis and machine learning tools will be evaluated in a collaboration with another research group at HFT Stuttgart.

Another open challenge is posed by the lack of proper a user and authorization management in openHAB, which has been already the subject of research $[4,19,33]$. Within the ongoing project, the requirement of public buildings to allow access to the smart system based on user roles (students, staff, facility management) will be addressed to some extent.

To enable adoption and further development of the proposed ideas and use cases by other universities and public institutions, they are documented and published as open-source, again [17]. Finally, a systematic evaluation of the prototypes will have to be carried out to conclude whether and under which circumstances the proposed smart home and IoT technologies can be considered as suitable for being used in public buildings.

\section{ACKNOWLEDGMENTS}

The authors thank the Federal Ministry of Education and Research of Germany (BMBF) for supporting the explorative research on Smart Public Buildings (13FH9E03IA) within the joint research project FH-Impuls 2016: iCity - Intelligente Stadt.

\section{REFERENCES}

[1] Mark Aberdour. 2007. Achieving Quality in Open-Source Software. IEEE Software 24, 1 (2007), 58-64. https://doi.org/10.1109/MS.2007.2

[2] Fadi Al-Turjman (Ed.). 2019. Security in IoT-enabled spaces. CRC Press, Boca Raton, FL. https://doi.org/10.1201/9780429031915

[3] Ahmed Abdulhasan Alwan, Andres Baravalle, Mihaela Anca Ciupala, and Paolo Falcarin. 2019. An Open Source Software Architecture for Smart Buildings. In Intelligent Systems and Applications, Kohei Arai, Supriya Kapoor, and Rahul Bhatia (Eds.). Advances in Intelligent Systems and Computing, Vol. 869. Springer International Publishing, Cham, 160-169. https://doi.org/10.1007/978-3-03001057-7\{_\}14

[4] Jochen Bauer, Michael Hechtel, Christoph Konrad, Martin Holzwarth, Hilko Hoffmann, Thomas Feld, Sven Schneider, Ingo Zinnikus, Andreas Mayr, and Jörg Franke. 2020. ForeSight - An AI-driven Smart Living Platform, Approach to Add Access Control to openHAB. https://doi.org/10.1007/978-3-030-51517-1_40

[5] Mark Church. 2019. Docker Reference Architecture: Designing Scalable, Portable Docker Container Networks. https://success.docker.com/article/networking

[6] Department for Communities and Local Government London. 2015. Display Energy Certificates: current regime and how it could be streamlined and improved. https://assets.publishing.service.gov.uk/government/uploads/system/ uploads/attachment_data/file/402703/Consultation_on_DEC_Regime.pdf

[7] DIN 18040-1:2010-10. 2010. DIN 18040-1:2010-10: Barrierefreies Bauen - Planungsgrundlagen - Teil 1: Offentlich zugängliche Gebäude. https://doi.org/10 $31030 / 1715500$

[8] Alexander Dobler. 2019. Adaption of the openHAB Smart Home System for Usage in Public Institutions with Multiple Buildings. Thesis. Hochschule für Technik Stuttgart, Stuttgart.

[9] Łukasz Dywicki. 2019. Community survey 2019. https://community.openhab. $\mathrm{org} / \mathrm{t} /$ community-survey-2019/73653

[10] Kenan Ercan. 2020. Passenger Flow Metering in a Smart Public Building. Thesis Hochschule für Technik Stuttgart, Stuttgart.

[11] European Commission. 2019. Energy performance of buildings. https://ec.europa eu/energy/en/topics/energy-efficiency/energy-performance-of-buildings
[12] European Commission. 2019. What is personal data? https://ec.europa.eu/info/ law/law-topic/data-protection/reform/what-personal-data_en

[13] European Union. 2016. Regulation (EU) 2016/679 of the European Parliament and of the Council of 27 April 2016 on the protection of natural persons with regard to the processing of personal data and on the free movement of such data, and repealing Directive 95/46/EC (General Data Protection Regulation) (Text with EEA relevance): GDPR. https://eur-lex.europa.eu/eli/reg/2016/679/oj

[14] Nicola Fiedler. 2017. Smart Public Building: Bestandsaufnahme, Analyse von Anforderungen und Anwendungspotentialen in öffentlichen Gebäuden am Beispiel der Stadt Stuttgart. Thesis. Hochschule für Technik Stuttgart, Stuttgart.

[15] Free Software Foundation Europe. 2019. Public Money, Public Code: Modernising Public Infrastructure with Free Software. https://download.fsfe.org/campaigns/ pmpc/PMPC-Modernising-with-Free-Software.pdf

[16] I. Georgievski, P. Gupta, and M. Aiello. 2019. Activity Learning for Intelligent Buildings. In 2019 IEEE 10th Annual Ubiquitous Computing, Electronics Mobile Communication Conference (UEMCON). 0916-0923.

[17] GitLab. 2019. Smart Public Building Repository. https://transfer.hft-stuttgart.de/ gitlab/smartpublicbuilding

[18] Stephan Günthner (Ed.). Mai 2017. Smart City Charta: Digitale Transformation in den Kommunen nachhaltig gestalten (stand: mai 2017 ed.). Bundesinstitut für Bau- Stadt- und Raumforschung (BBSR) im Bundesamt für Bauwesen und Raumordnung (BBR), Bonn.

[19] Florian Heimgaertner, Stefan Hettich, Oliver Kohlbacher, and Michael Menth. 06. - 09.06.2017. Scaling home automation to public buildings: A distributed multiuser setup for OpenHAB 2. In 2017 Global Internet of Things Summit (GIoTS). IEEE, 1-6. https://doi.org/10.1109/GIOTS.2017.8016235

[20] Hochschule für Technik Stuttgart. 2016. Hausordnung. http://www.hft-stuttgart. $\mathrm{de} /$ Studium/Studienorganisation/Weitere\%20Satzungen/Hausordnung

[21] Home Assistant. 2019. Releases. https://www.home-assistant.io/faq/release/

[22] Lin Hsien-Tang. 2013. Implementing Smart Homes with Open Source Solutions. 7, 4 (2013), 289-296.

[23] Open Energy Monitor. 2019. Monitoring energy via utility meter pulse output. https://learn.openenergymonitor.org/electricity-monitoring/pulsecounting/introduction-to-pulse-counting

[24] openHAB. 2020. The open Home Automation Bus (openHAB). https://www. openhab.org/docs/

[25] openHABian. 2019. openHABian - Hassle-free openHAB Setup. https://www. openhab.org/docs/installation/openhabian.html

[26] Jim Sinopoli. 2010. Smart buildings systems for architects, owners, and builders. Butterworth-Heinemann, Amsterdam.

[27] Statista. 2019. Haushaltsgröße in den EU-Ländern 2018. https://de.statista.com/ statistik/daten/studie/350573/umfrage/haushaltsgroesse-in-den-eu-laendern/

[28] Statista. 2019. Wohnfläche je Wohnung in Deutschland nach Bundesländern im Jahr 2018. https://de.statista.com/statistik/daten/studie/70111/umfrage/ wohnflaeche-pro-wohnung-je-bundesland-2007/

[29] Bernhard Stengel. 2015. Ethische Überlegungen zu Smart Home. International Review of Information Ethics 22 (2015), 92-100.

[30] The Things Network Region Stuttgart. 2017. Community Site. https://www. thethingsnetwork.org/community/region-stuttgart/

[31] Brian Thomas and Diane Cook. 2016. Activity-Aware Energy-Efficient Automation of Smart Buildings. Energies 9, 8 (Aug 2016), 624. https://doi.org/10.3390/ en 9080624

[32] VDI. 2019. VDI 3814 Blatt 1:2019-01. Building automation and control systems (BACS) - Fundamentals.

[33] Sebastian Werner, Frank Pallas, and David Bermbach. 2018. Designing Suitable Access Control for Web-Connected Smart Home Platforms. In Service-oriented computing - ICSOC 2017 workshops, Lars Braubach (Ed.). Lecture Notes in Computer Science, Vol. 10797. Springer, Cham, 240-251. https://doi.org/10.1007/9783-319-91764-1\{_\}19

[34] Wilting, Klaus and Brandmueller, Oliver. 2019. ESP32-Paxcounter, Github Repository. https://github.com/cyberman54/ESP32-Paxcounter 